\title{
A LEARNING AND SOCIAL MANAGEMENT SYSTEM - VERSION 3.0
}

\author{
Alvaro Figueira ${ }^{1}$, Luciana Oliveira ${ }^{2}$ \\ ${ }^{1}$ CRACS INESC TEC \& University of Porto (PORTUGAL) \\ ${ }^{2}$ ISCAP / IPP Polytechnic of Porto \& CRACS INESC TEC (PORTUGAL)
}

\begin{abstract}
Current Learning Management Systems generically provide virtual places to conduct interactions between students and educators. Chats, forums and other communication mechanisms usually are present in any LMS. In this paper, we propose a tool that can be embedded in any LMS that features some sort of hierarchical communication mechanisms. The proposed system is capable of depicting and analyzing online interactions in an easy to understand social graph. The vertex positioning algorithm is based on social network analysis statistics, taken from the collected interactions, and is able to smoothly present the temporal evolution in order to find communicational patterns and report them to the educator and the students.
\end{abstract}

Keywords: LMS, Social Media, Integration, SNA, Social Graph.

\section{INTRODUCTION}

Social media is being increasingly used not only for personal and recreational activities but also as a powerful means to harness communication in education. Social networks, most notably, Facebook, Twitter and Instagram are being used all over the world by students to exchange information between themselves and the teacher. Despite different peculiarities of each social network, all of them offer great advantages over the traditional well-known learning management system, like Moodle and Blackboard. The main advantages of these social media systems rely on their ubiquitous communication capabilities, as well as their state-of-the-art graphical interfaces, which greatly influence teenagers, but also adults, to adhere to their use [1]. It is clear that current learning management systems cannot compete with these communication technologies and should not even aim to compete for their use.

In this paper, we present a system which is capable to integrate the best of the two worlds: from one side, a system which is capable to store educational contents, to engage them in pedagogical activities and to keep track of their evolution; on the other side, the possibility to include advanced/instant messaging capacities, as well as mechanisms to easily adapt to different digital media formats. Current social network systems allow the exchange of photos, videos, audio, digital presentations (to name a few) in a very simple way. Actually, many of them allow this exchange in the middle of a chat. Clearly, there is an advantage to use these technologies on the pedagogical field as communication becomes much easier.

However, the system we present goes far from allowing students to communicate through social media. We integrate in it analytics from the use of these communication fields gathering important information about student performance, difficulties, their pace, communication patterns, generic metrics for the class and specific issues to each student.

In fact, we propose the creation of a "social graph" in Moodle, built automatically from the interactions between course participants, maintained on social media, which are tracked by the system, and the analytics associated with this graph. Namely, we identify the "connectivity degree", and the "betweenness" of each participant finding which is most influential, which acts as an information hub and how information propagates on this network. We expand the analytics finding connected components in the graph to understand how these virtual communities relate and work together.

In the next section, we present some considerations about the use of the theory of social network analysis in the context of education, focusing on the visual representation of communication in the class. In section three we briefly describe a system that bridges a learning management system with a social network to profit from the communication capabilities of social networks, while mining the interaction patterns in order to better understand the class. In section four we describe how a social graph can be useful in education and which network metrics it should use. As these requirements lead 
to intractable situations in graph drawing, we present our heuristics to mitigate the drawing problems, leading to the algorithm presented in section five. Finally, we present our conclusions in section six.

\section{SOCIAL NETWORK ANALYSIS IN EDUCATIONAL SETTINGS}

Social Network Analysis (SNA) [2] consists in the "mapping" and analysis of the relations between people, groups or organizations, through a visual representation and statistical analysis [3] of the implicit/explicit relations between them. The visual representation of these metrics result in a network that includes a set of actors that interact among themselves as well as information flows. The usual representation of social networks is a graph, in which actors are vertices and the relations between them are represented by lines/edges of the graph.

In educational settings, we are interested in analysing the communication patterns of the students (and the teacher). Therefore, we use the "interaction" as a relation between two nodes. In order to distinguish direct communications from broadcasts, which is typical in forums, we consider the event "reply to" a previous posted message as an interaction. The counting process of the answers that are received and sent begins at the first "reply to" in a discussion. The analysis process in SNA generally consists in three perspectives. The first reports to the actors' positions individually; the second, to group action and, the third to the group or community as a whole.

\subsection{A Social Graph to Depict Class Interactions}

The ego network created as described above can be depicted as a graph where each node is a different student (or the teacher) and a line between nodes represent a single communication between those two actors. If the we assign weights to these lines, then we can represent the number of interactions that occurred between the two actors. Accordingly, we must create the same graphical representation for every node and every interaction.

As it is clear to understand, for a class of reasonable dimension, this kind of representation can be confusing of nodes are not well positioned. For example, one simple heuristic for graph drawing states that whenever possible, two edges should not cross. However, in every network with more than 4 nodes this is no longer possible. Therefore, the layout is a serious concern in graph drawing. Nevertheless, even with very dense graph representations, the shape play an important role identifying communication patterns, as illustrated in Fig. 1.

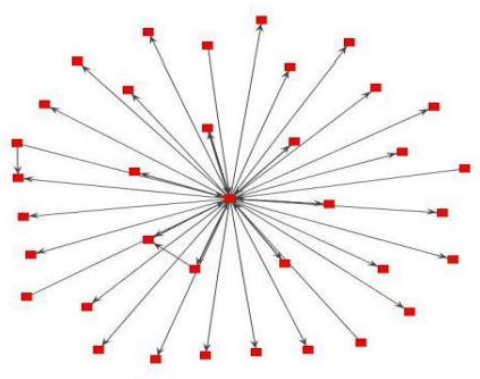

Answer Person

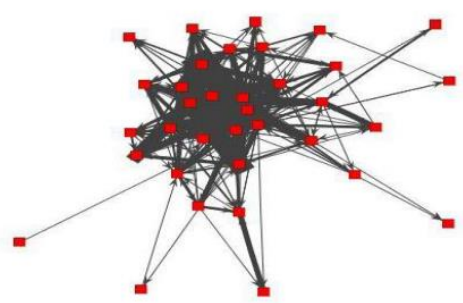

Discussion Person

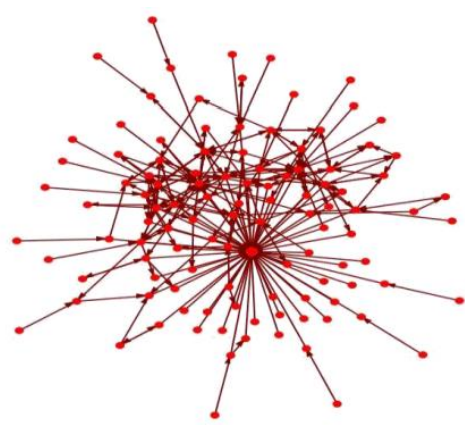

Reply Magnet

Fig. 1. Characterizing different types of ego networks.

\section{A REVIEW OF THE EDUBRIDGE SYSTEM}

Recently, it has been proposed a system that bridges one learning management system with a social network [4] - the Moodle and Facebook, respectively.

The proposed system is based on a set of Moodle blocks that share a communications engine and a database which is stored locally. The system's architecture is based on five modules. As a top layer, there is the communications module (googleoauther2) which establishes a connection between Moodle and Facebook through the Facebook's authentication mechanism, using the user's credentials. Then, the control is passed to the module fbgroups to retrieve the groups owned by the user. This module is also responsible for creating lists of users belonging to the groups and to set some counters for usage statistics. The module fbcomments retrieves all the posts and comments that 
were posted in timeline of the Facebook group. Finally, the modules fbstats and fbtotalstats present the local statistics, and global statistics, about the selected group or all the considered groups, respectively.

The choice to implement the functionalities using "Moodle blocks" was straightforward according to the authors: i) it's easier to keep and maintain the code modular; ii) Moodle blocks have the capabilities to include code which is centrally administrated, and which can share a single common access to tables in the LMS database; iii) the blocks positioning is easily customized.

\subsection{The EduBridge's blocks}

The EduBridge system aims at integrating Facebook groups in the Moodle environment. The proposed architecture is based on a model-view-controller and can be applied to most social networks with minimal effort. In this section the system and user interface are briefly presented with several examples taken from its real usage.

The system's interface is composed of a set of four Moodle blocks with which users, particularly teachers, can interact to display and manage information and analytics retrieved from Facebook groups in their Moodle Dashboard (known as "My home" up to Moodle's 2.8 version). This aims at framing the system in the most recent Moodle's philosophy for managing the teacher's educational workspace. The four blocks are designated as: "Facebook Groups", "Facebook Posts \& Comments", "Facebook Statistics" and "Total Statistics". The block's behaviour is interdependent, thought the information displayed on all four blocks is adjusted to users' interaction with the main block "Facebook Groups".

The "Facebook Groups" block retrieves the list of groups that the authenticated user manages on Facebook. This means that all educational and personal groups are displayed and are accessible through the Moodle's interface. This is the main block among the four-set because it allows teachers to arrange the information and views to be generated in the remaining blocks, by selecting the group or set of groups that he / she wishes to analyse.

Through the block "Facebook Posts and Comments" (Fig. 2) the system provides the teacher with the ability to access all the group's posts and interactions on the central area of the Moodle's Dashboard. This block's main purpose is to increase the level of integration between the two platforms, since it eliminates the need to access a second environment (Facebook) in order to check updates and new user's interactions, which may or may not require action.

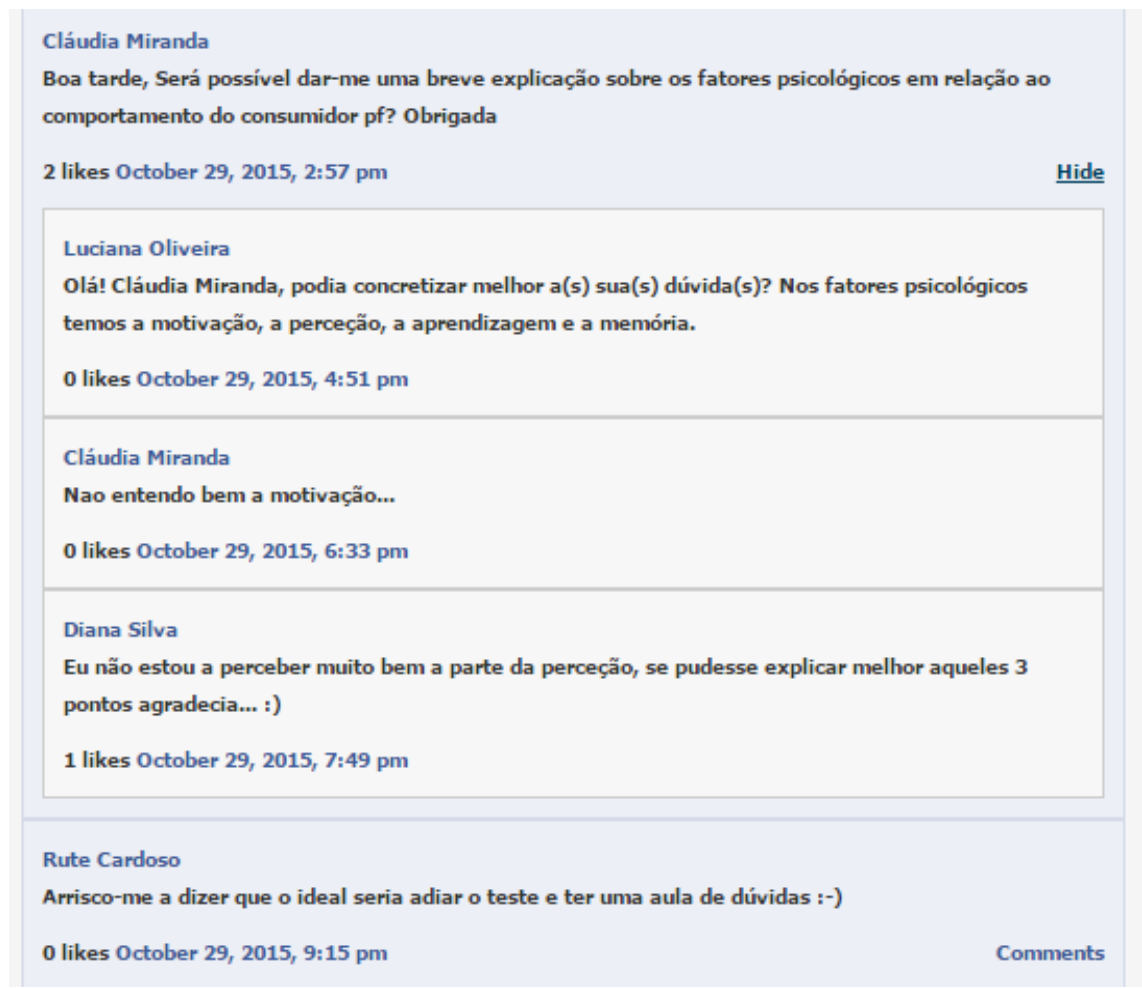

Fig. 2 "Facebook Posts and Comments" block with expanded comments view ([4]). 
The "Group Statistics" block provides teachers with statistical data concerning the group's total number of posts, total number of posts, median of comments per post, retrieves the group's most recent post and comment and also the most popular post, which is the post with more likes, as illustrated in Fig. 3. This block also displays user interaction statistics, providing relevant information for student's performance assessment, as illustrated in Fig. 4.

In the aforementioned block, the statistical information about student's performance is organized in two sections: "Users who posted" new messages in the group and the corresponding number of likes; and "Users who commented", that is, the users who interacted with the messages posted on the same group, by posting replies of liking comments.

Users (teacher and students) are ranked by the amount of interaction they have generated in the group. Rankings of users' statistical data are particularly useful during the teaching-leaning process and specifically relevant for student continuous or final assessment.

For instance, a user may post a lot of messages in the group, but may not receive feedback at all (that is, the content may not be relevant for that community). The user may not interact at all with other users' messages (that is, he / she may not find other user's content relevant and / or this may be an indicator of lack of interest, inexistence of sense of belonging in the group, etc.).

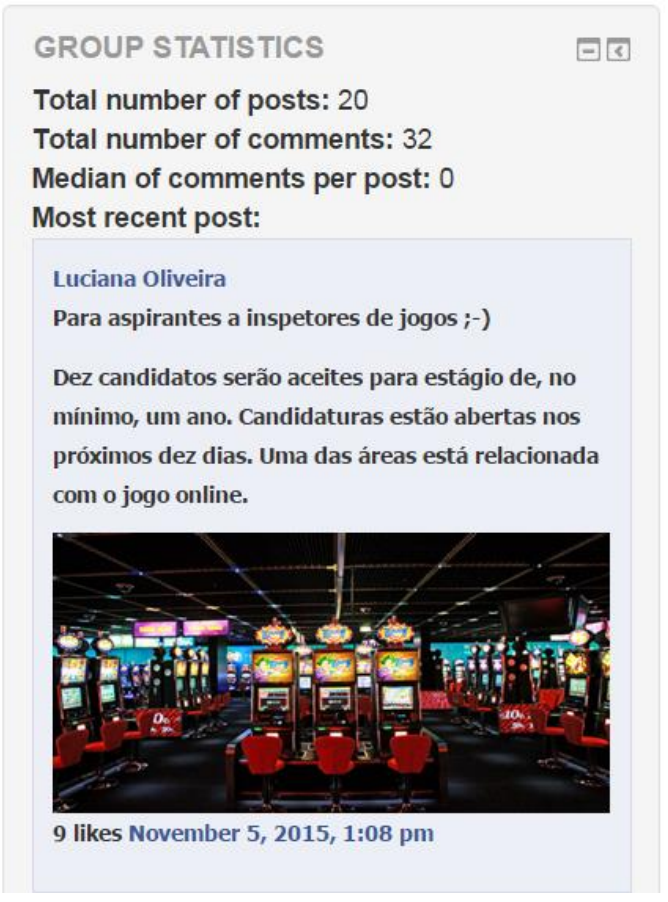

Fig. 3 Group Statistics module ([4]).

On the other hand, a user may not introduce any content at all in the group, but he / she may be an active and valuable member to the community, either by commenting, replying to comments (i.e., fostering discussion) or liking his / her colleagues' messages. It is up to the teacher to consider the weight of these parameters in assessing individual or group performance (if assessment is applicable).

The fourth block, "Facebook Total Statistics", illustrated in Fig. 4 a) and b), displays the global statistics for the Moodle course selected on the "Facebook Groups" block. In case two or more Facebook groups are assigned to the same Moodle course, this block will provide the teacher with the aggregated statistics for that set of groups. This feature is particularly relevant when the teacher intends to have the class divided into groups and uses several Facebook groups to guide and support each group separately.

This block is also useful for teachers needing aggregated statistics for all his / her Facebook groups or for personalized sets of groups, which are matched in the "Facebook Groups" block. 


FACEBOOK TOTAL STATISTICS
Comunicação de Marketing
Total number of posts: 40
Total number of likes: 155
Total number of comments: 156
Total number of CLikes: 92
Group with recent changes:
IPT P22D (15/16)
Course groups:
IPT - P21D (14/15)
IPT P22D (15/16)
Users who posted:
Luciana Oliveira

Fig. 4 (a) Facebook Total Statistics (b) User posting statistics.

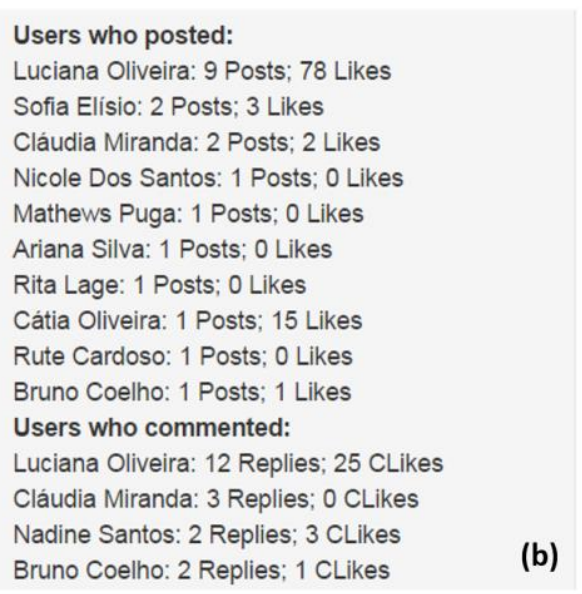

(b)

The integration and interaction between the illustrated blocks provides teachers with a valuable tool for analysing learning communities based on Facebook groups which have emerged as privileged environments for discussions, much in the same line as forums, however, with much more flexibility

A very relevant detail, which is not frequently mentioned in literature, and is of the utmost importance in this scenario, is the discrepancy between the pace of research and its concrete / tangible corresponding transfer into the educational context. In some organizational cultures, the proneness to adopt or adapt to new pedagogical methodologies / technologies is very slow. This was recognized by researchers during the initial stages of introduction of institutional LMS in education [5], when teachers' proneness to adopt technology mediated education was a hot topic. Therefore, the decision to build upon the existing Moodle architecture aims at addressing the real needs of current teachers and students, whose main element in technology mediated education is still the traditional LMS.

Learning analytics is a current hot topic which the presented system aims to address on the teacher and student perspective, in order to provide a validated tool for formal and informal learning assessment. However, the analytics presented by the system do not incorporate any of the features provided SNA, which are fundamental in a social context. In fact, the integration of SNA, for instance in the form of social graphs, is crucial given the social nature of the environment under analysis. The metrics and visualizations provided by SNA are of particular relevance when analysing users' interactions, which consist of the main input / output of the learning process in social media based learning communities.

\section{VISUALIZING A SOCIAL GRAPH}

Educational insights on graph interaction analysis provide teachers with knowledge on which type of community has developed or is being developed. Many considerations could be built on this topic: whether the interactions should be teacher or student centred, which are not our main purpose. We can however stress that knowledge on this aspect is both relevant for teachers and for students, namely: to identify the type of community, its main intervenient, the origin of interactions and how oneself relates to the others in that group.

Traces of activity left by social media users can facilitate perceptions on individual behaviour, social relationships, and community efficiency [6]. Tools and processes to analyse social traces are essential for enabling educators to study and nurture meaningful and sustainable social interactions occurring in learning communities.

\subsection{Characteristics of the Social Graph}

Where there are nodes with more connections and connected with denser parts of the network, those nodes (ie, students) allow the information to propagate faster, and make the network more responsive to posts. Mapping this situation to the academic world and students, this means that these students are indispensable to have success in communicating to the whole class. This empiric reasoning has a theoretical base on some SNA metrics, namely the in and out degree of a node (number of connections of a node, in each way) [7], the connectivity of the network and the betweenness of a node. While the connectivity of the network can be expressed in the number of edges or nodes that 
need to be removed in order to disconnect the network, being a measure of resilience (which is important, for example for MOOCs), the betweenness centrality if a measure applied to a single node. It expresses the number of shortest paths that include the node. Frequently this metric is employed as a measure of power and popularity.

\subsection{Visualization Limitations}

Clearly, the simple visual effects used until now, namely in the EduBridge system, like node size, line width, node and line colors are not enough to describe all these important properties. The main drawing concern is where to place the nodes in a two-dimensional space? And, which kind of global layout to use? Traditional layout algorithms use force-placement heuristics where each node tens to repel another node unless it is connected through an edge. However, other parameters, like centralization properties must be taken into consideration (example, placing the most important node in the center of the graph).

The optimal placement of nodes is a complex problem which may be intractable for a network of considerable size (in the order of hundreds of nodes). Therefore, many "shortcuts" in the form of heuristics must be used to cut the inherent complexity of this procedure. Nevertheless, an easy-tounderstand graph must depend on three factors: i) the defined visual properties, ii) the layout algorithm and, iii) filters to reduce the amount of data, in order to allow clear inspection of a subset of the network. Usually these parameters should be tuned in a recursive way, as illustrated in Fig. 5.

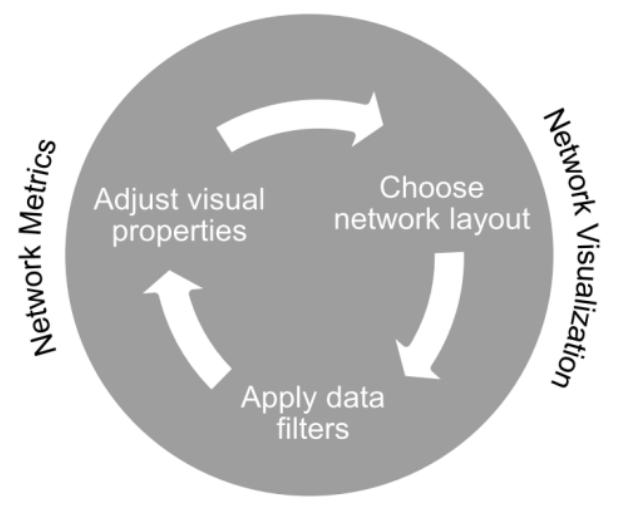

Fig. 5. Creating a human-understandable social network graph.

\section{AUTOMATICALLY DRAWING A SOCIAL GRAPH}

We propose a tool to help characterizing online interactions depicting them in the form of a graph that in turn is built with the help of "social network analysis" indicators. The proposed graph represents all interactions occurred up to the drawing moment. This characteristic allows building a "history" of interactions, drawing each network state in a singular time-frame. A slideshow of all available frames can then provide additional insight for the teacher as she may analyze the class according to different key moments and observe its progress over time, such as actors that maintain leadership roles during most of the time, or actors that shift between more or less active positions in different moments.

We stress that the motivation for adding history capabilities is to understand how the online community evolves along time. Although a graphical representation of a specific state of an online community is of much use, that representation lacks the temporal dimension which may hide important aspects of interaction that occurred in past.

There is a vast literature and research area concerning automatic graph drawing [8]. A variety of layout algorithms that are based on graph theoretical foundations have been developed in the last three decades [9]. In the sixties' Tufte wrote a paper on how to draw a graph [10] in which he suggested an algorithm that places each vertex into the center of its neighbors. A long way of research has been pursued since then. However, some basic criteria, supported by psychology studies [11][12], still hold: vertices displaying the objects should not overlap each other, nor the lines representing the edges. Moreover, one would want to minimize the edge crossings.

Our algorithm evolves from a set of basic principles/premises to improve readability and ease of understanding: a) distribute vertices avoiding overlapping; b) information hubs should tend to be 
placed near the center; c) minimize the crossing of edges and of vertices; d) group cliques; e) dense net tends to spread equally.

According to those principles, we built a model of "orbits" in which we place the vertices equally spaced in clockwise manner. The outer orbit is placed near the border of the drawing canvas. In Fig. 6 we depict this model.

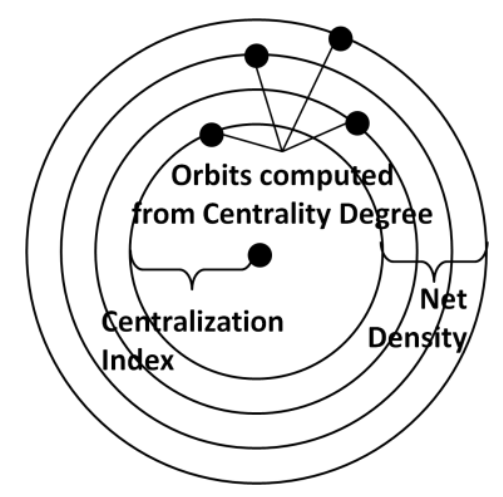

Fig. 6 Vertex positioning model.

The orbit of each vertex is computed according to its centrality degree (the greater the degree, the closer to the center will be the orbit). The centralization index is useful to compute the radius of the closest orbit to the center (a centralization index of $100 \%$ means that the smallest orbit will have radius of 0 ). The net density parameter is useful to set the number of possible orbits (a dense net, will have more orbits). We present the drawing algorithm in Listing 1 where we define $k$-clique as a clique with $k$ vertices, and an object as either a weak-connected component, a clique or a vertex.

Listing 1. Vertex positioning algorithm.

1. WCC Detection: identify all weak-connected components of size $\geq 3$

2. WCC Reduction: pairs of wcc of size $n$, sharing $n-1$ vertices are treated as a single wwc logicallyconnected by their vertex with higher betweenness

3. Let objects $\leftarrow W C C \cup V$

4. Orbit assignment: for each obj in objects

4.1. compute its orbit:

4.1.1.if $o b j$ is a vertex compute its normal centrality degree

4.1.2.if $o b j$ is a $k$-clique compute $\frac{\sum C_{i}^{C D}}{k}$, where $C_{i}^{C D}$ is the $\mathrm{CD}$ of vertex $i$

4.1.3. if $o b j$ is a wcc

5. Layout: dispose objects clockwise, equally spaced

6. Vertex Permutation: for each obj find a permutation $(P)$ of its vertices that minimizes distance to other vertices outside the $o b j: \min \left\{\sum \operatorname{distances}\left(P_{i}\right)\right\}$

This algorithm can be computed in real-time (up to some hundreds of nodes) and allows a smooth visual transition between different time frames (characterized by new interactions). Moreover, it organizes connected components and higher-order nodes to facilitate the visualization and intuitive analysis of the class interactions.

\section{CONCLUSIONS}

Nowadays, the trend in Learning Management Systems is no longer focused in creating more features or novel activities. New generation of LMS are evolving into cloud systems and using open API for resource integration. Social Media are slowly being integrated but many other services too. The "piazza" system for managing QA and notes is a good example of a communication-oriented service that is being developed outside a LMS, but many more exist. More than ever it is important to manage and enforce student communication, but now it also is time to gain insight from the communication patterns in itself. 
We believe that the visualization of the construction, structure, dimension and evolution of the interaction's network is of high relevance for teachers and for students. A time span visualization as well as the network current status may allow teachers to identify risks and opportunities in community development, such as marginalized or low performance students, but also about high performing students that require additional challenging tasks, both aimed at preventing frustration and / or dropout. For students, the visualization of their position in the network, as well as rankings-based analytics could serve self-assessment and performance benchmarking tools. The effects of such tools on motivation to learn and improve student performance or to foster dropout are, however, not clear at the moment and lack extensive research, which we believe will be possible to conduct in a near future.

\section{ACKNOWLEDGEMENTS}

This work is supported by the ERDF - European Regional Development Fund through the COMPETE Programme (operational programme for competitiveness) and by National Funds through the FCT (Portuguese Foundation for Science and Technology) within project «Reminds/ UTAP-ICDT/EEICTP/0022/2014».

\section{REFERENCES}

[1] Oliveira, L. Social Student Relationship Management in Higher Education: extending educational and organisational communication into Social Media. in 9th Annual International Technology, Education and Development Conference. 2015. Madrid, Spain: IATED.

[2] Scott, J.: Social Network Analysis: a Handbook. Sage, London: Sage (1997)

[3] Borgatti, S. P., Everett, M. G.: Network Analysis of 2-Mode Data. Social Networks, pp. 243--269. http://www.analytictech.com/borgatti/papers/borgatti\%20-\%20network\%20analysis\%20of\%202mode\%20data.pdf. (1997) (accessed: September 2016).

[4] Oliveira, L. and Á. Figueira. EduBridge Social: Bridging Social Networks and Learning Management Systems. in 8th International Conference on Computer Supported Education: CSEDU. 2016. Italy: Scitepress.

[5] Rogers, E. M. (2010). Diffusion of innovations, Simon and Schuster.

[6] Hansen, D.L., et al. Do You Know the Way to SNA?: A Process Model for Analyzing and Visualizing Social Media Network Data. in Social Informatics (Sociallnformatics), 2012 International Conference on. 2012. IEEE.

[7] Freeman, L. C.: Centrality in Social Networks: Conceptual Clarification. Social Networks, 1, 215239. http://moreno.ss.uci.edu/27.pdf. (1978) (accessed: September 2016)

[8] Jünger, M., Mutzel, P.: Graph Drawing Software. Springer (2004)

[9] Nishizeki, T., Rahman, S.: Planar Graph Drawing. Lecture Notes Series on Computing, vol. 12, World Scientific (2004)

[10]Tufte, W.T.: How to Draw a Graph. Proceedings of the London Mathematics Society, vol. 13, pp. 743--768 (1963)

[11]Purchase, H.: Which aesthetic has the greatest effect on human understanding? In: G. Di Battista (ed) Graph Drawing'97, LNCS, vol. 1353, pp. 248--261. Springer (1997)

[12] Purchase, H., Allder, J.-A., Carrington, D.: User preference of graph layout aesthetics: A UML study. In: J. Marks (ed) Graph Drawing'00, LNCS, vol. 1984, pp. 5--18. Springer (2001) 